\title{
EDITORIAL
}

\section{Special Issue: Polymer surfaces, interfaces and thin films}

Polymer Journal (2016) 48, 323; doi:10.1038/pj.2016.26

$\mathrm{T}$ The chemical and physical properties of polymers at interfaces should be closely related to the manifestation of their functions. For example, the performance of polymer-based organic solar cells strongly depends on the structure and dynamics of the conjugated polymers at various interfaces. Biocompatibility of polymers is also correlated with the polymer behavior at the interface with a body fluid. The importance of studying polymer interfaces can be seen not only in the examples above but also in many other polymer applications. So far, researchers in various fields have reported that the structural and physical properties of polymers at interfaces are not the same as those in the corresponding bulk phase. However, a clear understanding of why that must be is still lacking. Thus, as a benchmark effort, it has been strongly desired to clarify controlling factors in such polymer behaviors at interfaces. To realize this, studies geared towards the relation of fundamental sciences of polymers at interfaces with the performance of polymer thin devices are needed.

Recently, Polymer Journal has started to publish a special issue focusing on hot topics in polymer science, ${ }^{1-6}$ at least once a year. They have been very successful and cited by researchers not only in polymer science but also in various disciplines. Thus, in this special issue, our editorial team solicited researchers to present theoretical, experimental and computational studies on the following topics: synthesis, structure, physical properties and function of polymers at interfaces. To realize this, the Editor-in-Chief, Prof. Takashi Kato, organized a team composed of three associate editors, Profs. Keiji Tanaka, Takeshi Serizawa and Wen-Chang Chen, and a guest editor, Prof. Kookheon Char. We eventually could collect 22 original articles, 5 focus reviews and 1 review from outstanding researchers over Asia. The contents in articles and reviews are intriguing and very high in the scientific level.
This leads to our self-confidence that this issue will make a great success as before. Finally, we would like to express our sincere gratitude to all authors and referees for contributing greatly to this special issue.

Keiji Tanaka ${ }^{1}$, Takeshi Serizawa ${ }^{2}$, Wen-Chang Chen $^{3}$, Kookheon Char $^{4}$ and Takashi Kato ${ }^{5}$

${ }^{1}$ Department of Applied Chemistry, International Institute for Carbon-Neutral Energy Research (WPI-I2CNER), Kyushu University, Fukuoka, Japan; ${ }^{2}$ Department of Organic and Polymeric Materials, Tokyo Institute of Technology, Tokyo, Japan; ${ }^{3}$ Department of Chemical Engineering, Institute of Polymer Science and Engineering, National Taiwan University, Taipei, Taiwan; ${ }^{4}$ Interdisciplinary Program in Nano-Science and Technology and NANO Systems Institute, School of Chemical and Biological Engineering, Seoul National University, Seoul, Korea and ${ }^{5}$ Department of Chemistry and Biotechnology, School of Engineering, The University of Tokyo, Tokyo, Japan E-mail: k-tanaka@cstf.kyushu-u.ac.jp

1 Kato, T., Aoshima, S. \& Kikuchi, H. Special issue: self-assembled materials. Polym. J. 44, 451 (2012).

2 Asakura, T. \& Asano, A. NMR of polymers: recent advances and innovations. Polym. J. 44, 733 (2012).

3 Kanaya, T., Sakurai, K. \& Takahara, A. Special issue: application of quantum beams to polymer science and engineering. Polym. J. 45, 2 (2013).

4 Venanzi, M. \& Kimura, S. Special issue: peptide materials. Polym. J. 45, 467 (2013).

5 Serizawa, T. Special issue: biorelated polymers and materials. Polym. J. 46, 435 (2014).

6 Kato, T., Kikuchi, H. \& Imai, H. Special issue: fusion materials: creative development of materials and exploration of their function through molecular control. Polym. J. 47, 77 (2015). 